\title{
Planetary transit candidates in Corot-IRa01 field ${ }^{\star}$
}

S. Carpano ${ }^{1}$, J. Cabrera ${ }^{2,3}$, R. Alonso ${ }^{4}$, P. Barge ${ }^{4}$, S. Aigrain ${ }^{6}$, J.-M. Almenara ${ }^{7}$, P. Bordé ${ }^{8}$, F. Bouchy ${ }^{9}$, L. Carone ${ }^{10}$, H. J. Deeg ${ }^{7}$, R. De la Reza ${ }^{11}$, M. Deleuil ${ }^{4}$, R. Dvorak ${ }^{12}$, A. Erikson ${ }^{2}$, F. Fressin ${ }^{22}$, M. Fridlund ${ }^{1}$, P. Gondoin ${ }^{1}$, T. Guillot ${ }^{13}$, A. Hatzes ${ }^{14}$, L. Jorda ${ }^{4}$, H. Lammer ${ }^{15}$, A. Léger ${ }^{8}$, A. Llebaria ${ }^{4}$, P. Magain ${ }^{16}$, C. Moutou ${ }^{4}$, A. Ofir ${ }^{20}$, M. Ollivier ${ }^{8}$, E. Janot-Pacheco ${ }^{21}$, M. Pätzold ${ }^{10}$, F. Pont ${ }^{6}$, D. Queloz ${ }^{5}$, H. Rauer ${ }^{2}$, C. Régulo ${ }^{7}$, S. Renner ${ }^{2}, 17,18$, D. Rouan ${ }^{19}$, B. Samuel ${ }^{8}$, J. Schneider ${ }^{3}$, and G. Wuchterl ${ }^{14}$

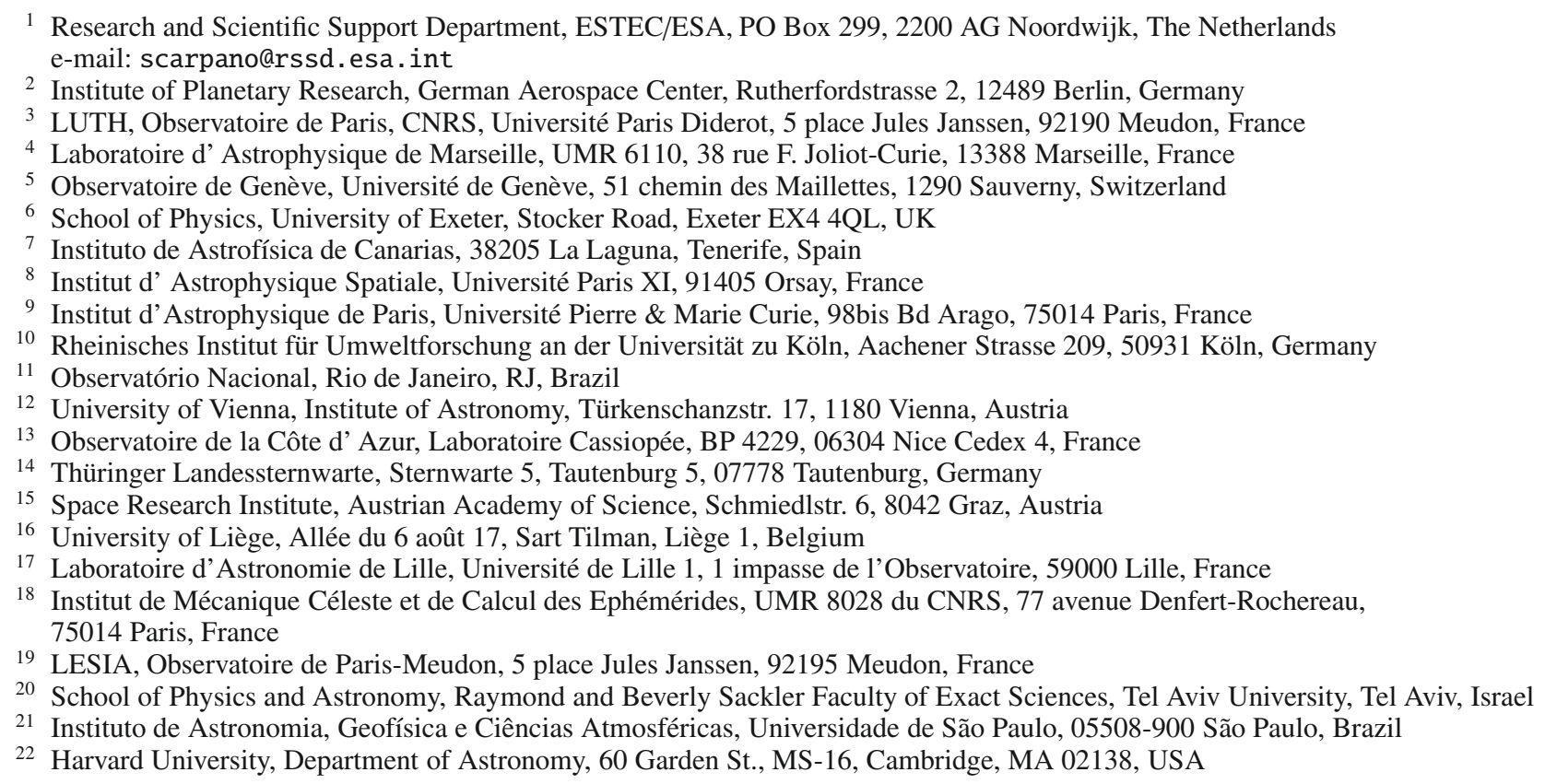

Received 19 February 2009 / Accepted 27 July 2009

\section{ABSTRACT}

Context. CoRoT is a pioneering space mission devoted to the analysis of stellar variability and the photometric detection of extrasolar planets.

Aims. We present the list of planetary transit candidates detected in the first field observed by CoRoT, IRa01, the initial run toward the Galactic anticenter, which lasted for 60 days.

Methods. We analysed 3898 sources in the coloured bands and 5974 in the monochromatic band. Instrumental noise and stellar variability were taken into account using detrending tools before applying various transit search algorithms.

Results. Fifty sources were classified as planetary transit candidates and the most reliable 40 detections were declared targets for follow-up ground-based observations. Two of these targets have so far been confirmed as planets, CoRoT-1b and CoRoT-4b, for which a complete characterization and specific studies were performed.

Key words. stars: planetary systems - techniques: photometric - binaries: eclipsing - planetary systems

\footnotetext{
* The CoRoT space mission, launched on December 27th 2006, has been developed and is operated by CNES, with contributions from Austria, Belgium, Brazil, ESA, Germany, and Spain. Four French laboratories associated with the CNRS (LESIA, LAM, IAS ,OMP) collaborate with CNES on the satellite development. First CoRoT data are available to the public from the CoRoT archive:

http://idoc-corot.ias.u-psud.fr.
}

\section{Introduction}

The transit method for detecting exoplanets identifies candidates by monitoring stars for long periods of time, then processing the data to isolate stars that exhibit a periodic flux drop consistent with a Jupiter-sized or smaller companion passing between its parent star and the observer. A large number of targets is necessary, because the probability of a planet producing an observable transit is very low, due to geometric effects. The processing and analysis of gathered data is thus a major undertaking. 
The methodology used to analyse thousands of light curves in the search for transiting extrasolar planets was described in detail by Gould et al. (2006) for OGLE data. We summarize here a few concepts:

- CoRoT light curves are processed and filtered for instrumental noise as described in Drummond et al. (2008);

- each of the detection teams applies its own algorithms for detrending the signal (e.g., variability, noise) and searching for planetary transits (see Moutou et al. 2005, 2007);

- the results of each team are combined and each candidate is discussed individually.

In our final discussion, a check is performed to reject clear eclipsing binaries, i.e., systems with lights curves that exhibit secondary eclipses, out-of-transit photometric modulations, and/or events that are too deep to be caused by transiting planets. The shape of transits is also analysed: photometric dips of planets have a "U" shape, while binaries are more "V" shaped. These criteria, however, can only be used for data of with relatively high signal-to-noise ratios. Some examples of eclipsing binary light curves are shown in Figs. 1-3. Raw light curves are shown in the top panel and smoothed, detrended, and folded light curves are shown in the bottom panel. These exhibit the typical features of small secondary eclipses, in phase modulation, and secondary transits out of phase 0.5 . Figure 4 shows the raw and folded light curves of a good planetary candidate with a shallow transit (source No. 46, E2 4124, in Tables 2 and 3).

Source confusion with background binaries will also produce false candidates; this is true in particular for CoRoT because of its large PSF (Barge et al. 2008b; Drummond et al. 2008). In this case, one benefits from the three coloured bands of the CoRoT photometric mask. When a candidate is bright enough for its flux to be separated into three bands/colours, the transit is occasionally not observed in one or more of the bands/colours or is a significantly different depth in the separate bands/colours. For the remaining candidates, photometric and/or spectroscopic follow-up are essential to determining of the masses of the system components, by measurements of the radial velocity shift of the spectral lines of the parent star that occur as the planet orbits. In the case of CoRoT, photometric follow-up is useful in cases of source confusion. Spectroscopic ground-based measurements, on the other hand are essential for determinating the masses of the system components, via measurements of the radial velocity shift of the spectral lines of the parent star that occur as the planet orbits.

In this work, we present the results of the joint work of the CoRoT detection teams, a huge effort to separate the wheat from the chaff to provide accurate parameters for the interesting objects. The IRa01 CoRoT data are now public. We offer the fruits of our labor to the astronomical community so it may serve as a starting point for interested researchers. Section 2 contains some details about this initial CoRoT run, including the candidate information from the satellite itself. In Sect. 3, we provide the list of the 50 transiting candidates observed in the first CoRoT field IRa01 and their transit parameters. Results are summarized in Sect. 4.

\section{CoRoT observations of IRa01 field}

CoRoT observed its first field from early February 2008 until early April, for approximatively 60 days. The run code "IRa01" is explained as following. The "IR" means "initial run" in contrast to the subsequent "long runs" (LR) and "short runs" (SR).
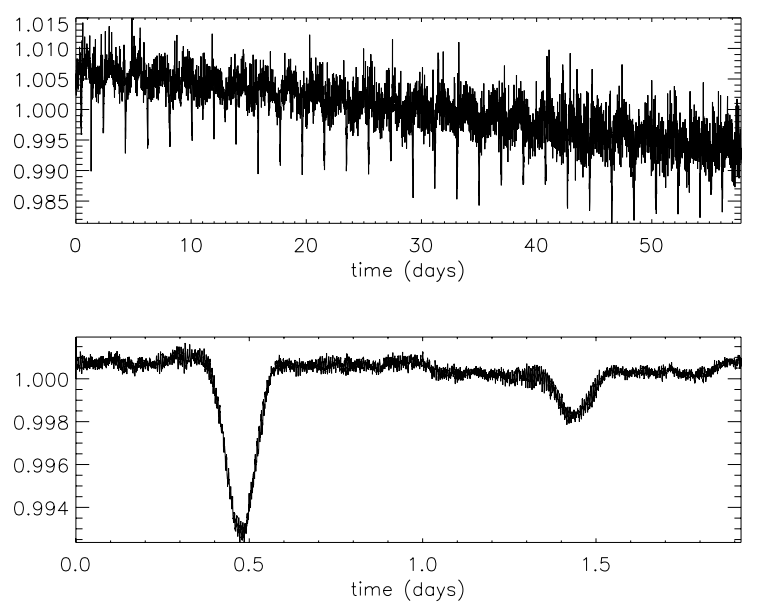

Fig. 1. An eclipsing binary found in IRa01 showing small secondary eclipses. Raw (top), smoothed, and detrended folded light curve (bottom).
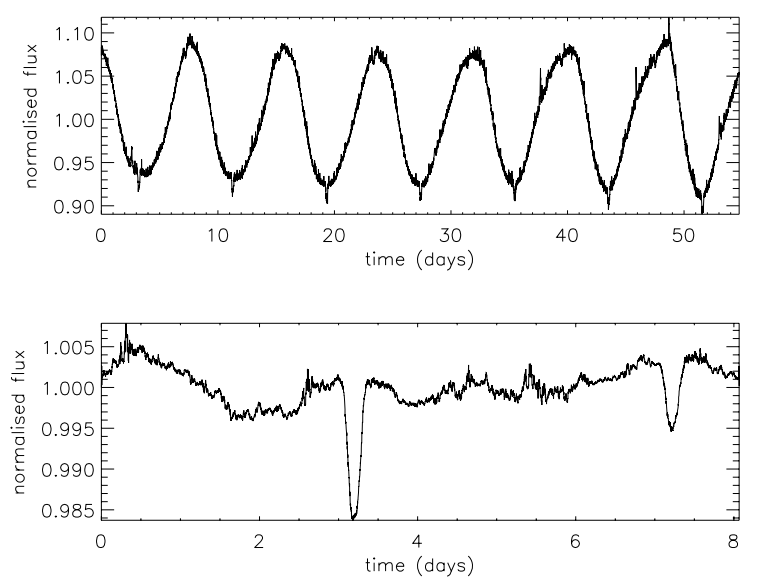

Fig. 2. An eclipsing binary found in IRa01 showing in phase modulation. Raw (top), smoothed, and detrended folded light curve (bottom).
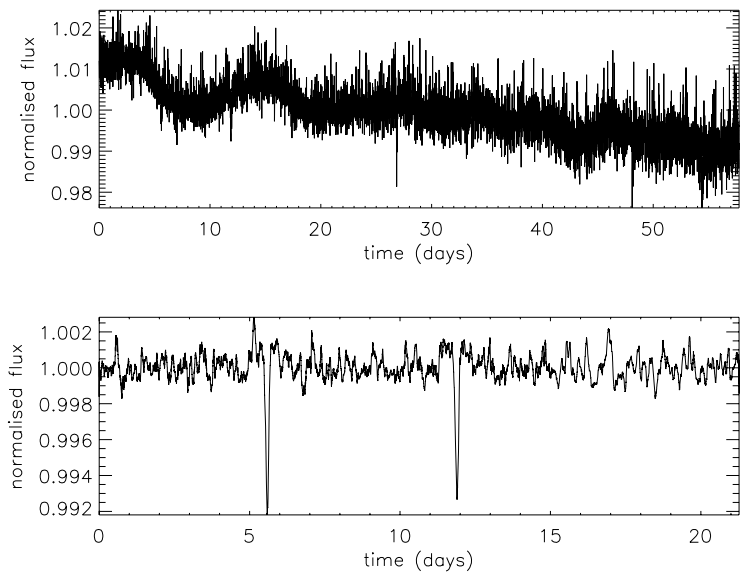

Fig. 3. An eclipsing binary found in IRa01 showing orbital eccentricities. Raw (top), smoothed, and detrended folded light curve (bottom).

The third letter refers to the direction with respect to the Galactic center ("a", as in this case, anticenter or "c" Galactic center). The last two digits are the sequence for this type of observation (01 being the first one). 

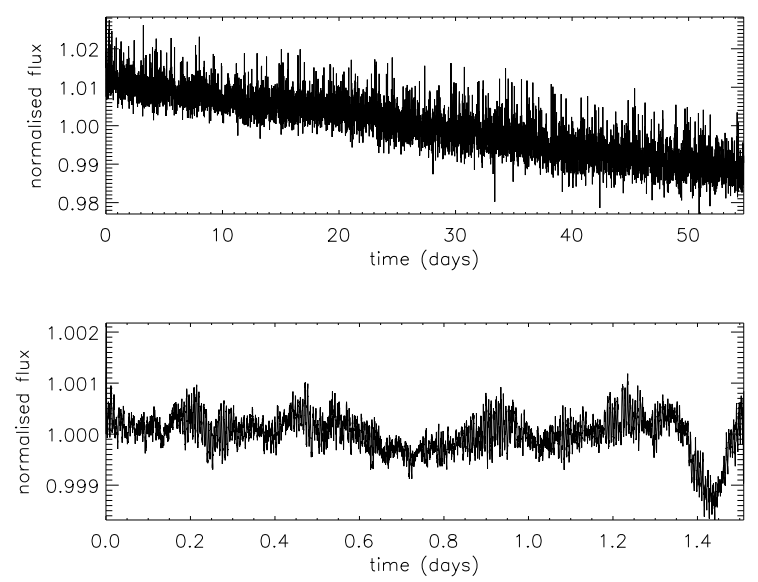

Fig. 4. Raw and folded light curve of a planetary candidate (source No. 46, E2 4124).

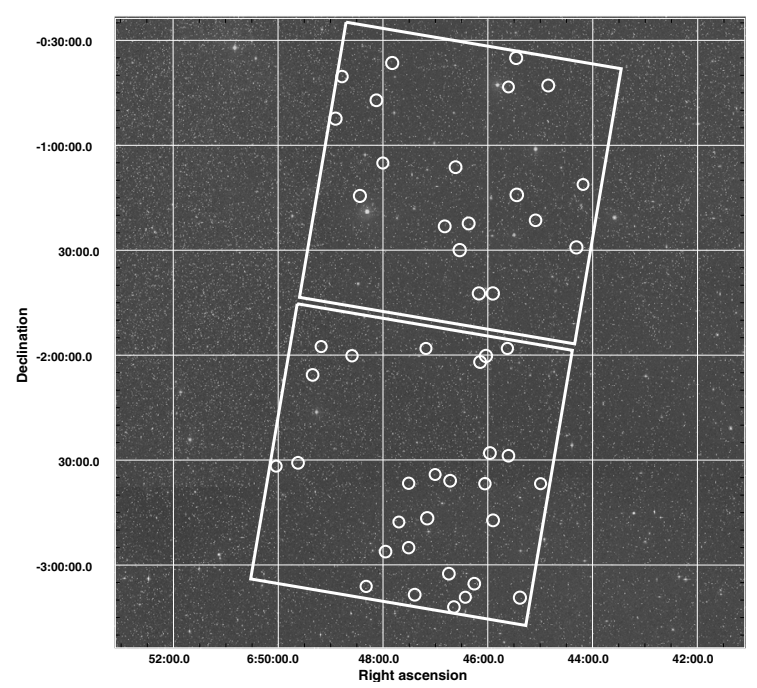

Fig. 5. DSS image of the sky observed by CoRoT during the IRa01. Overlaid are the positions of the 50 planetary transit candidates and the portion of the field covered by the 2 exoplanets CCD.

Table 1. List of the detection teams (institutes and people).

\begin{tabular}{cl}
\hline \hline Team & Participants \\
\hline DLR & Heike Rauer, Anders Erikson, Stefan Renner \\
ESTEC & Malcolm Fridlund, Stefania Carpano \\
Exeter & Suzanne Aigrain, Frédéric Pont, Aude Alapini \\
IAC & Hans Deeg, José M. Almenara, Clara Régulo \\
IAS & Pascal Bordé, Benjamin Samuel \\
Köln & Martin Pätzold, Ludmilla Carone \\
LAM & Pierre Barge, Roi Alonso \\
LUTh & Jean Schneider, Juan Cabrera \\
\hline
\end{tabular}

3898 sources were observed in IRa01 using coloured filters ( $B, V, R$ colours), while 5974 sources were monitored at a single monochromatic band. To analyse these data sets, detection teams were established in a number of different collaborating institutes. Their task is to provide a list of candidates, their ranking (according to the probability of their planetary nature), as well as a first estimate of transit ephemerides and parameters. At this point, 8 teams are participating, each using their own independently-developed detection methods. Table 1 contains a list of the different institutions involved and the names of the contributors. Some of these methods were presented during a pre-launch performance simulation described in Moutou et al. (2005), while others have been or will be developed in separated papers (i.e., Carpano \& Fridlund 2008; Renner et al. 2008; Régulo et al. 2007). The algorithms described in these works are generally based on the following fundamental approaches: correlation with sliding transit template, box-shaped signal search, box-fitting least-squares (BLS), wavelet transformation, or Gaussian fitting of folded light curve. A merged list of 92 planetary transit candidates was compiled by the teams, which was reduced to a final list of 50 candidates after discussion (most of the other 42 candidates were classified as binaries). The 40 most robust candidates were recommended for ground-based follow-up, the results of which are reported in Moutou et al. (2009). Two planets from the final list of 40 candidates, CoRoT-1b and CoRoT-4b, have so far been confirmed as planets. More details about the discovery of these two planets can be found in Barge et al. (2008a) and Aigrain et al. (2008), respectively.

Figure 5 shows the sky coverage of the 2 CCDs dedicated to exoplanetary science and the positions of the candidates within this field of view. Table 2 provides a list of planetary candidates in the IRa01 field, including their CoRoT- and window-ID numbers, J2000 positions, an indication of whether the candidate was observed in three colours ("CHR") or monochrome ("MON"), magnitude(s), and exposure times (in s). A change in the time sampling from $512 \mathrm{~s}$ to $32 \mathrm{~s}$ indicates that several transits were detected in the first portion of the light curve and the Alarm Mode (Quentin et al. 2006; Surace et al. 2008) was chosen to resample those targets to improve the time accuracy. All parameters derived from the Exo-Dat database (Meunier et al. 2007; Deleuil et al. 2009) .

\section{Compiling a list of candidates with their transit parameters}

The selection process of planetary candidates for follow-up has several steps. First, each detection team analyses the tens of thousands of light curves independently using their own filtering and detection codes. A list of candidates is compiled by each team, and arranged in order of a numerical priority from 1 for the best candidates to 3 or 4 for doubtful sources (e.g., "V" shaped transit, suspicion of secondary transits, noisy data, mono-transits). A "B" is given for binary sources. All lists are then merged into a single list, where the sources at the top level are the candidates found by several teams at high priorities. The teams interact regularly by means of weekly teleconferences. Apart from most likely candidates and the binaries, all sources are rediscussed and reanalysed. The list of transit candidates selected by the detection teams and sorted by the probability of their planetary nature of highest probability is then examined by the follow-up teams. They are responsible for confirming (or rejecting) the planetary nature of each candidate by ground-based observations. They focus primarily on the candidates of highest priorities, although stellar magnitude and amount of observing time available will influence their final decisions.

The transit parameters of the candidates were estimated as follows. First, a low-order polynomial was fit to the regions around each transit in an attempt to normalise the data. A first estimate of the period and epoch are used to phase-fold the light curve. The data points are binned, errors being assigned according to the standard deviation of the points inside each bin divided by the square-root of the number of points in each bin. 
Table 2. List of the 50 planetary transit candidates detected in the CoRoT IRa01 field, see text for more details.

\begin{tabular}{|c|c|c|c|c|c|c|}
\hline No. & CoRoT-ID & Win-ID & Right ascension, declination & Exo-Dat $R$ mag & Colour & Time Sampling \\
\hline 1 & 0102723949 & E1 2046 & $6: 44: 11.03943,-1: 11: 13.236$ & 13.60 & CHR & 512 \\
\hline 2 & 0102729260 & E1 1319 & $6: 44: 18.72070,-1: 29: 11.328$ & 14.77 & $\mathrm{CHR}$ & 512 \\
\hline 3 & 0102763847 & E1 1158 & $6: 45: 5.28076,-1: 21: 25.236$ & 13.11 & $\mathrm{CHR}$ & 512,32 \\
\hline 4 & 0102787048 & E1 0288 & $6: 45: 36.24023,-0: 43: 17.400$ & 13.17 & CHR & 512,32 \\
\hline 5 & 0102787204 & E2 3787 & $6: 45: 36.48010,-2: 28: 50.664$ & 14.00 & CHR & 512 \\
\hline 6 & 0102798247 & E2 1857 & $6: 45: 53.99963,-2: 47: 15.900$ & 14.06 & CHR & 512,32 \\
\hline 7 & 0102806520 & E1 4591 & $6: 46: 10.31982,-1: 42: 23.688$ & 13.70 & CHR & 512 \\
\hline 8 & 0102809071 & E2 1136 & $6: 46: 15.36072,-3: 5: 19.608$ & 13.20 & CHR & 512,32 \\
\hline 9 & 0102815260 & E2 2430 & $6: 46: 25.68054,-3: 9: 13.284$ & 14.57 & CHR & 512,32 \\
\hline 10 & 0102825481 & E2 0203 & $6: 46: 43.20007,-2: 35: 58.308$ & 13.07 & CHR & 512,32 \\
\hline 11 & 0102826302 & E2 1712 & $6: 46: 44.63928,-3: 2: 32.208$ & 13.98 & CHR & 512,32 \\
\hline 12 & 0102829121 & E1 0399 & $6: 46: 49.44031,-1: 23: 11.616$ & 13.66 & CHR & 512 \\
\hline 13 & 0102855534 & E2 1736 & $6: 47: 30.47974,-2: 55: 4.116$ & 13.85 & CHR & 512,32 \\
\hline 14 & 0102856307 & E1 0396 & $6: 47: 31.68091,-1: 23: 26.808$ & 13.58 & CHR & 512 \\
\hline 15 & 0102874481 & E2 1677 & $6: 47: 57.11975,-2: 56: 10.896$ & 13.84 & CHR & 512 \\
\hline 16 & 0102890318 & E2 1126 & $6: 48: 19.20044,-3: 6: 7.776$ & 13.43 & CHR & 512,32 \\
\hline 17 & 0102895957 & E1 0783 & $6: 48: 26.40015,-1: 14: 31.344$ & 12.72 & CHR & 512,32 \\
\hline 18 & 0102912369 & E1 0330 & $6: 48: 46.79993,-0: 40: 21.972$ & 13.45 & CHR & 512,32 \\
\hline 19 & 0102918586 & E1 2755 & $6: 48: 54.23950,-0: 52: 22.800$ & 12.24 & CHR & 512,32 \\
\hline 20 & 0102753331 & E1 4617 & $6: 44: 50.87952,-0: 42: 53.280$ & 15.87 & MON & 512 \\
\hline 21 & 0102759638 & E2 3724 & $6: 44: 59.52026,-2: 36: 45.144$ & 14.79 & MON & 512 \\
\hline 22 & 0102777119 & E2 4290 & $6: 45: 23.04016,-3: 9: 23.688$ & 15.03 & MON & 512 \\
\hline 23 & 0102779966 & E1 4108 & $6: 45: 26.87988,-1: 14: 9.456$ & 14.94 & MON & 512 \\
\hline 24 & 0102780627 & E1 1531 & $6: 45: 27.83936,-0: 35: 4.668$ & 14.98 & MON & 512 \\
\hline 25 & 0102788073 & E2 2009 & $6: 45: 37.67944,-1: 58: 9.300$ & 14.18 & MON & 512 \\
\hline 26 & 0102798429 & E1 2774 & $6: 45: 54.23950,-1: 42: 22.752$ & 15.52 & MON & 512 \\
\hline 27 & 0102800106 & E2 3010 & $6: 45: 57.59949,-2: 28: 0.732$ & 15.49 & MON & 512 \\
\hline 28 & 0102802430 & E2 4300 & $6: 46: 2.16064,-2: 0: 13.428$ & 14.33 & MON & 512 \\
\hline 29 & 0102802996 & E2 3150 & $6: 46: 3.35999,-2: 36: 46.548$ & 14.96 & MON & 512 \\
\hline 30 & 0102805893 & E2 2604 & $6: 46: 8.88062,-2: 2: 0.348$ & 15.42 & MON & 512 \\
\hline 31 & 0102812861 & E1 2648 & $6: 46: 21.84082,-1: 22: 19.128$ & 15.59 & MON & 512 \\
\hline 32 & 0102819021 & E1 2328 & $6: 46: 32.16064,-1: 29: 58.812$ & 15.10 & MON & 512 \\
\hline 33 & 0102821773 & E1 4998 & $6: 46: 36.95984,-1: 6: 15.768$ & 15.48 & MON & 512 \\
\hline 34 & 0102822869 & E2 4058 & $6: 46: 38.88062,-3: 12: 4.860$ & 15.55 & MON & 512 \\
\hline 35 & 0102835817 & E2 3425 & $6: 47: 0.23987,-2: 34: 7.140$ & 15.60 & MON & 512 \\
\hline 36 & 0102841669 & E2 3854 & $6: 47: 9.36035,-2: 46: 39.108$ & 14.91 & MON & 512 \\
\hline 37 & 0102842120 & E2 3952 & $6: 47: 10.07996,-2: 57: 1.944$ & 13.98 & MON & 512 \\
\hline 38 & 0102842459 & E2 1407 & $6: 47: 10.79956,-1: 58: 7.356$ & 14.77 & MON & 512 \\
\hline 39 & 0102850921 & E2 2721 & $6: 47: 23.75977,-3: 8: 32.424$ & 12.90 & MON & 512 \\
\hline 40 & 0102855472 & E2 0704 & $6: 47: 30.47974,-2: 36: 40.140$ & 13.86 & MON & 512 \\
\hline 41 & 0102863810 & E2 4073 & $6: 47: 42.00073,-2: 47: 43.404$ & 15.39 & MON & 512 \\
\hline 42 & 0102869286 & E1 2329 & $6: 47: 49.44031,-0: 36: 29.052$ & 15.52 & MON & 512 \\
\hline 43 & 0102876631 & E1 3336 & $6: 48: 0.23987,-1: 5: 5.964$ & 14.56 & MON & 512 \\
\hline 44 & 0102881832 & E1 4911 & $6: 48: 7.43958,-0: 47: 9.024$ & 15.05 & MON & 512 \\
\hline 45 & 0102903238 & E2 4339 & $6: 48: 35.52063,-2: 0: 12.096$ & 15.61 & MON & 512 \\
\hline 46 & 0102926194 & E2 4124 & $6: 49: 3.59985,-2: 48: 34.488$ & 15.73 & MON & 512 \\
\hline 47 & 0102932089 & E2 3819 & $6: 49: 10.79956,-1: 57: 34.452$ & 16.00 & MON & 512 \\
\hline 48 & 0102940315 & E2 4467 & $6: 49: 20.63965,-2: 5: 37.716$ & 15.81 & MON & 512 \\
\hline 49 & 0102954464 & E2 3856 & $6: 49: 37.43958,-2: 30: 49.140$ & 15.97 & MON & 512 \\
\hline 50 & 0102973379 & E2 1063 & $6: 50: 2.40051,-2: 31: 47.604$ & 14.09 & MON & 512 \\
\hline
\end{tabular}

A Levenberg-Marquardt algorithm (Levenberg 1944; Marquardt 1963 ) is used to fit a trapezoid (where its center, depth, duration, and time of ingress are the fit parameters) to the phase-folded curve. The best-fit model trapezoid is then cross-correlated at each individual transit in the light curve, to determine their centers. A linear fit to the resulting $\mathrm{O}-\mathrm{C}$ diagram refines the estimations of the period and epoch. With this new ephemeris, the process is iterated, until the ephemerides are within the error bars of the previous values (typically one iteration is sufficient). The error in both the period and epoch are the formal errors in the linear fit.
Table 3 lists the transit parameters for the 50 planetary candidates: identifiers, coordinates, periods, and epochs with their associated errors, the transit duration (in hours) and depth (in \%), and an estimate of the stellar density inferred by the transit light curve fit as explained in Seager \& Mallén-Ornelas (2003). This parameter combined with the other characteristics of the candidates (e.g., depth, duration, shape, out of transit modulation, stellar parameters) are used as input for the ranking of candidates given to the follow-up team. We note that the light curve of the candidate 37 is contaminated with the curve of a clear eclipsing binary (source 97 in Table A.1). The value of its transit parameters may therefore have been affected. 
Table 3. Transit parameters of the 50 planetary candidates.

\begin{tabular}{|c|c|c|c|c|c|c|c|c|}
\hline No. & "Win-ID & Period (d) & Error period $(\mathrm{d})$ & $\begin{array}{r}\text { Epoch }(\mathrm{d}) \\
+2454000\end{array}$ & "Error epoch $(\mathrm{d})$ & 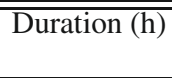 & 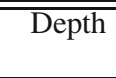 & 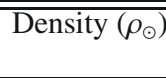 \\
\hline 1 & E1 2046 & - & - & 167.9153 & $2.1 \mathrm{E}-03$ & 5.638 & $1.2 \mathrm{E}-02$ & - \\
\hline 2 & E1 1319 & 1.69851 & $2.6 \mathrm{E}-05$ & 136.4886 & 8.5E-04 & 2.335 & 4.4E-03 & 0.5374 \\
\hline 3 & E1 1158 & 10.53096 & $1.3 \mathrm{E}-04$ & 140.0856 & 3.3E-04 & 2.159 & $1.4 \mathrm{E}-02$ & 8.9253 \\
\hline 4 & E1 0288 & 7.89296 & 4.6E-04 & 135.0691 & 2.3E-03 & 4.512 & $3.7 \mathrm{E}-03$ & 0.3740 \\
\hline 5 & E2 3787 & 0.85809 & $3.9 \mathrm{E}-06$ & 138.1467 & 1.4E-04 & 2.660 & $1.3 \mathrm{E}-03$ & 0.1955 \\
\hline 6 & E2 1857 & 0.82169 & $1.4 \mathrm{E}-05$ & 138.3168 & $6.6 \mathrm{E}-04$ & 1.829 & 4.7E-03 & 0.8132 \\
\hline 7 & E1 4591 & 4.29539 & $3.5 \mathrm{E}-05$ & 136.6231 & 2.6E-04 & 2.295 & $2.9 \mathrm{E}-03$ & 1.0074 \\
\hline 8 & E2 1136 & 1.22387 & $3.4 \mathrm{E}-06$ & 139.2401 & 1.3E-04 & 2.748 & $1.8 \mathrm{E}-03$ & 0.2076 \\
\hline 9 & E2 2430 & 3.58747 & 9.2E-05 & 139.2341 & 8.4E-04 & 5.570 & $1.2 \mathrm{E}-02$ & 0.5040 \\
\hline 10 & E2 0203 & 5.16868 & $1.9 \mathrm{E}-05$ & 138.7811 & $1.2 \mathrm{E}-04$ & 2.918 & $3.4 \mathrm{E}-02$ & 5.3662 \\
\hline 11 & E2 1712 & 2.76741 & $5.8 \mathrm{E}-05$ & 139.6140 & 5.3E-04 & 4.114 & $2.4 \mathrm{E}-03$ & 0.6866 \\
\hline 12 & E1 0399 & 33.06200 & $3.5 \mathrm{E}-03$ & 151.7875 & 2.2E-03 & 3.015 & $1.5 \mathrm{E}-02$ & 11.0820 \\
\hline 13 & E2 1736 & 21.72025 & $1.5 \mathrm{E}-03$ & 144.2915 & $2.8 \mathrm{E}-03$ & 13.175 & $1.2 \mathrm{E}-02$ & 0.1613 \\
\hline 14 & E1 0396 & 7.82394 & $6.9 \mathrm{E}-04$ & 140.0779 & 2.7E-03 & 2.788 & $8.2 \mathrm{E}-04$ & 0.6066 \\
\hline 15 & E2 1677 & - & - & 156.8022 & $1.2 \mathrm{E}-03$ & 6.795 & $3.0 \mathrm{E}-02$ & - \\
\hline 16 & E2 1126 & 1.50900 & $1.2 \mathrm{E}-05$ & 138.3265 & 3.3E-04 & 2.450 & $2.2 \mathrm{E}-02$ & 2.7151 \\
\hline 17 & E1 0783 & - & - & 162.9538 & $1.3 \mathrm{E}-03$ & 5.498 & $6.4 \mathrm{E}-03$ & - \\
\hline 18 & E1 0330 & 9.20191 & $3.4 \mathrm{E}-04$ & 141.3652 & $1.3 \mathrm{E}-03$ & 4.404 & $1.2 \mathrm{E}-02$ & 3.1234 \\
\hline 19 & E1 2755 & 4.39125 & 4.2E-05 & 139.3811 & 3.9E-04 & 2.486 & $2.4 \mathrm{E}-02$ & 3.8575 \\
\hline 20 & E1 4617 & 19.75581 & $3.8 \mathrm{E}-03$ & 143.8531 & $3.1 \mathrm{E}-03$ & 16.595 & $4.0 \mathrm{E}-02$ & 0.1324 \\
\hline 21 & E2 3724 & 12.32616 & $1.4 \mathrm{E}-03$ & 142.4015 & 2.7E-03 & 11.802 & $1.0 \mathrm{E}-02$ & 0.1094 \\
\hline 22 & E2 4290 & 2.20546 & $1.5 \mathrm{E}-05$ & 139.6775 & 2.1E-04 & 8.741 & $4.0 \mathrm{E}-03$ & 0.0307 \\
\hline 23 & E1 4108 & 7.36644 & 8.4E-04 & 137.9420 & $2.7 \mathrm{E}-03$ & 2.911 & $5.2 \mathrm{E}-03$ & 1.6112 \\
\hline 24 & E1 1531 & 2.38147 & 6.7E-05 & 137.2002 & 8.8E-04 & 2.160 & $1.2 \mathrm{E}-02$ & 2.0203 \\
\hline 25 & E2 2009 & 10.84581 & $1.4 \mathrm{E}-03$ & 141.7762 & $2.9 \mathrm{E}-03$ & 5.040 & 4.1E-03 & 0.2817 \\
\hline 26 & E1 2774 & 1.60551 & $5.9 \mathrm{E}-05$ & 135.8954 & $1.3 \mathrm{E}-03$ & 3.214 & 7.2E-03 & 0.3168 \\
\hline 27 & E2 3010 & 23.20918 & $6.1 \mathrm{E}-03$ & 159.0750 & $2.6 \mathrm{E}-03$ & 3.182 & $1.7 \mathrm{E}-02$ & 9.3127 \\
\hline 28 & E2 4300 & 5.80656 & 3.7E-04 & 139.0526 & $1.6 \mathrm{E}-03$ & 3.236 & $5.1 \mathrm{E}-03$ & 0.8538 \\
\hline 29 & E2 3150 & - & - & 163.4256 & 2.1E-03 & 4.041 & 1.7E-02 & - \\
\hline 30 & E2 2604 & 3.81967 & $1.4 \mathrm{E}-04$ & 138.2163 & $1.3 \mathrm{E}-03$ & 4.366 & $2.5 \mathrm{E}-03$ & 0.2051 \\
\hline 31 & E1 2648 & 3.68241 & $2.5 \mathrm{E}-04$ & 138.4929 & $1.8 \mathrm{E}-03$ & 3.138 & 8.8E-03 & 0.7965 \\
\hline 32 & E1 2328 & 4.50975 & $3.1 \mathrm{E}-04$ & 137.6290 & 2.1E-03 & 5.911 & $6.8 \mathrm{E}-03$ & 0.1238 \\
\hline 33 & E1 4998 & 10.08309 & $1.1 \mathrm{E}-03$ & 142.3704 & $2.6 \mathrm{E}-03$ & 2.787 & $1.9 \mathrm{E}-02$ & 5.6972 \\
\hline 34 & E2 4058 & - & - & 188.9298 & 4.2E-03 & 3.651 & 9.3E-03 & - \\
\hline 35 & E2 3425 & 1.18553 & 3.0E-05 & 139.2383 & 9.1E-04 & 2.996 & 3.6E-03 & 0.2295 \\
\hline 36 & E2 3854 & 1.14181 & 3.0E-05 & 138.9064 & 7.4E-04 & 1.971 & $1.4 \mathrm{E}-03$ & 1.4261 \\
\hline 37 & E2 3952 & 13.47756 & 4.1E-03 & 160.6192 & $3.2 \mathrm{E}-03$ & 2.605 & 2.2E-03 & 7.4800 \\
\hline 38 & E2 1407 & 5.16776 & 3.2E-04 & 140.9219 & $1.8 \mathrm{E}-03$ & 1.604 & $2.5 \mathrm{E}-02$ & 16.2410 \\
\hline 39 & E2 2721 & 0.61161 & $6.9 \mathrm{E}-06$ & 138.5715 & $3.5 \mathrm{E}-04$ & 2.569 & $6.0 \mathrm{E}-03$ & 0.4197 \\
\hline 40 & E2 0704 & 2.15520 & $6.1 \mathrm{E}-05$ & 139.2776 & 7.9E-04 & 5.891 & 7.2E-03 & 0.1898 \\
\hline 41 & E2 4073 & 15.00128 & $1.3 \mathrm{E}-03$ & 140.1756 & $2.4 \mathrm{E}-03$ & 5.347 & $3.9 \mathrm{E}-02$ & 2.7669 \\
\hline 42 & E1 2329 & 1.86725 & 5.6E-05 & 135.5561 & $5.8 \mathrm{E}-04$ & 2.636 & $3.7 \mathrm{E}-03$ & 0.4757 \\
\hline 43 & E1 3336 & 1.38972 & 3.3E-05 & 135.8757 & 7.7E-04 & 2.751 & $1.7 \mathrm{E}-03$ & 0.2017 \\
\hline 44 & E1 4911 & 2.16638 & 8.7E-05 & 136.5974 & $1.3 \mathrm{E}-03$ & 5.891 & 9.6E-03 & 0.1008 \\
\hline 45 & E2 4339 & 1.36204 & $3.9 \mathrm{E}-05$ & 139.1842 & $9.9 \mathrm{E}-04$ & 2.220 & $1.7 \mathrm{E}-03$ & 0.3215 \\
\hline 46 & E2 4124 & 1.50872 & 7.0E-05 & 139.5222 & $1.3 \mathrm{E}-03$ & 3.350 & 2.1E-03 & 0.1436 \\
\hline 47 & E2 3819 & 1.56554 & 4.7E-05 & 138.7047 & 8.6E-04 & 3.204 & 2.1E-02 & 0.6651 \\
\hline 48 & E2 4467 & 16.44935 & 2.2E-03 & 140.8322 & $3.0 \mathrm{E}-03$ & 5.527 & 1.4E-02 & 0.9068 \\
\hline 49 & E2 3856 & 16.56276 & $1.7 \mathrm{E}-03$ & 145.6439 & 2.7E-03 & 1.482 & $2.1 \mathrm{E}-02$ & 70.9060 \\
\hline 50 & E2 1063 & - & - & 171.7411 & $1.5 \mathrm{E}-03$ & 8.554 & 7.7E-03 & - \\
\hline
\end{tabular}

Figure 6 shows the transit depth versus orbital period for all sources in IRa01 including planetary candidates and stellar binaries. There does not seem to be any correlation between transit depth and period, for periods below 10 days. The correlation of the depth with the number of observed transits is evident for period $>10$ days. We note that several mono-transits have also been reported. This suggests that the detection methods used by the detection teams do not strongly depend on the number of transits as long as several are detectable. A detailed study of the capabilities of the detection algorithms is currently ongoing.

Figure 7 shows the same diagram but for the transit depth versus the $V$ magnitude. There is again no strong dependence between these two parameters that is apparent for magnitudes brighter than 16, a slight dependence is however evident for fainter stars. This might indicate that the noise is not dominated by photon noise but rather by instrumental effects, including hot pixels (see Fig. 8).

Hot pixels are characterized by sudden jumps in the light curve, followed either by an exponential or sudden decay. They are caused by high-energy particle impacts, mainly protons, on the detector. A description of the radiation effects on the CoRoT CCD can be found in Pinheiro da Silva et al. (2008). The number of hot pixels of intensity higher than a certain quantity of electrons at the beginning of the first 5 CoRoT runs (IRa01, SRc01, 


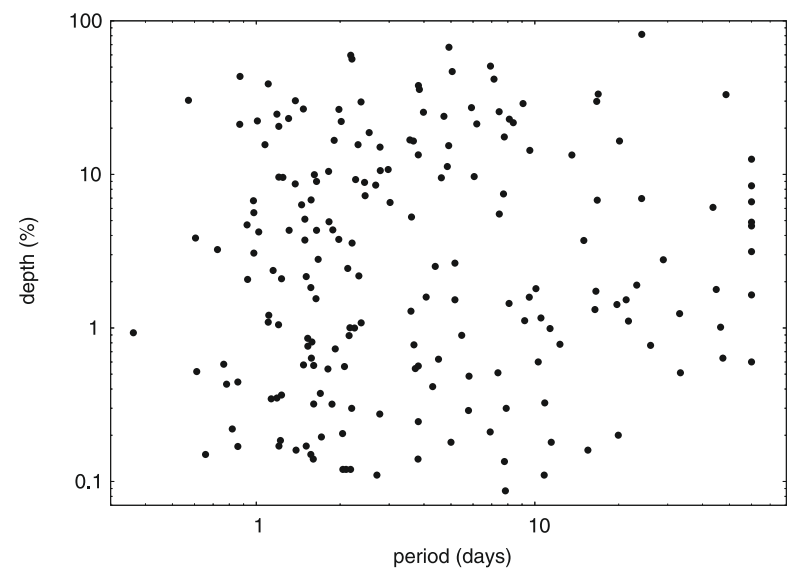

Fig. 6. Transit depth versus orbital period for all sources with detected transits (planetary candidates and clear stellar binaries).

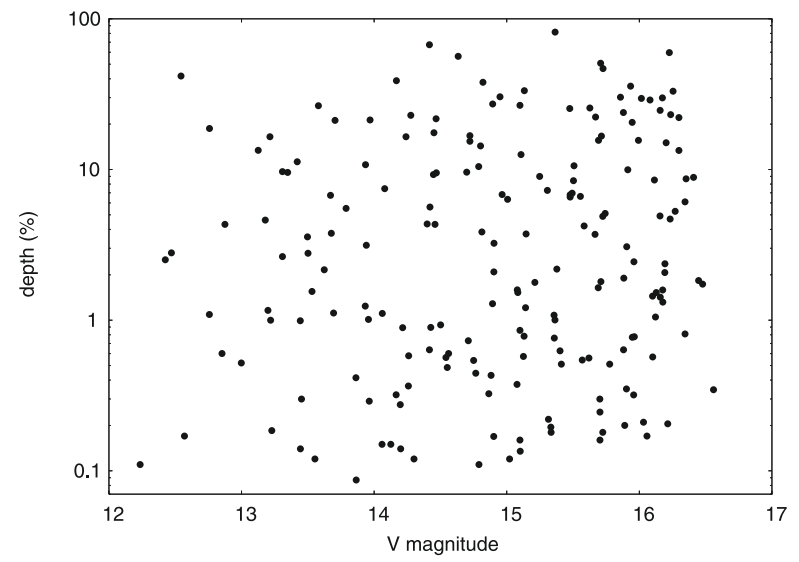

Fig. 7. Transit depth versus $V$ magnitude for all sources with detected transits (planetary candidates and clear stellar binaries).

LRc01, LRa01, and SRa01) are shown by Auvergne et al. (2009) in their Fig. 6. In the case of the initial run, about 26700, 3200, and 24 bright pixels were reported with an intensity of electrons higher than $300 \mathrm{e}^{-}, 1000 \mathrm{e}^{-}$, and $10000 \mathrm{e}^{-}$, respectively. No efficient filtering method has so far been found that is capable of removing these sudden jumps/decays from the light curves while leaving the transits intact. The detection teams deal with them mainly by renormalising the light curve before and after the jumps and leaving a gap at the place of the discontinuities. Replacing hot pixel events with short gaps avoids the detection of spurious signals without having a large impact on the detected transits.

A study of the noise properties was performed by Aigrain et al. (2009). They claim that, after pre-processing of the light curves to minimize long-term variations and outliers, the behaviour of the noise on a $2 \mathrm{~h}$ timescale is close to pre-launch specification. However, a noise level of a factor 2-3 above the photon noise is still found because of the residual jitter noise and hot pixel events. Furthermore, there is evidence of a slight degradation in the performance over time for the first 3 long runs (IRa01, LRc01, and LRa01).

The transit detection threshold is discussed in Moutou et al. (2009), following the model described in Pont et al. (2006). In Moutou et al. (2009), they examine the location of planet candidates in the magnitude versus transit signal $\left(d n^{0.5}\right.$, where $d$ is the transit depth and $n$ is the number of points in the transit). They find that the detection threshold does not

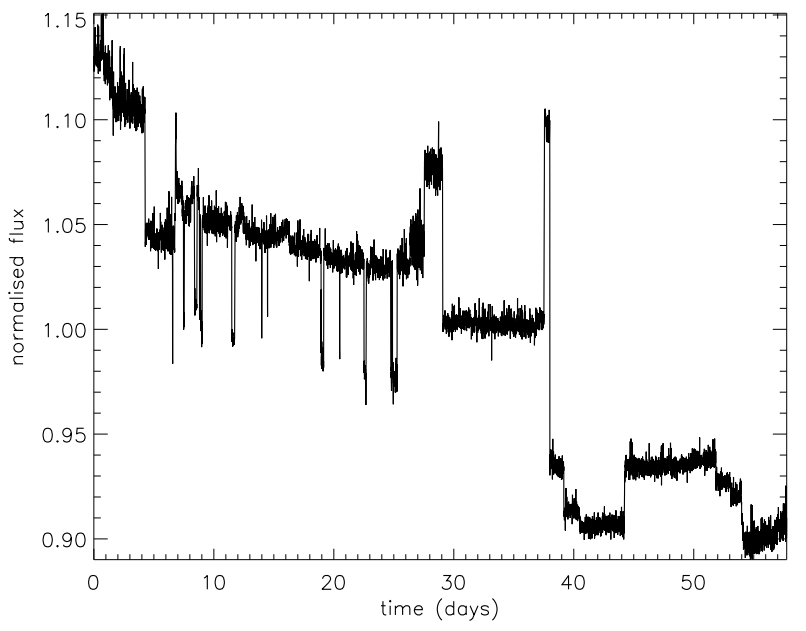

Fig. 8. Typical light curve containing frequent jumps caused by "hot pixels".

depend on magnitude and conclude that correlated fluctuations (instrumental effects or stellar variability) dominates, which is similar to what we conclude from Fig. 7. The detection limit is at $d n^{0.5}=0.009$, substantially higher than in the pre-launch models.

The implications of these noise properties and detection threshold on planet detection are discussed in Fressin et al. (in prep.). They use the CoRoTlux transit survey simulator described in Fressin et al. (2007) to show that the CoRoT yield on the first 4 fields is less than one-half that expected. This gap will probably be reduced as the follow-up of CoRoT candidates nears completion. Fressin et al. (2007) provides an estimate of the planet occurrence in close orbit around F-G-K dwarf stars as a function of the radius of the planet, which agrees with radial velocity, ground-based transit, and CoRoT discoveries. Interestingly, they show that CoRoT's detection of one Super-Earth (i.e., CoRoT-7b, see Léger et al. 2009) agrees with the high expectations from the HARPS team for the number of close-in Super-Earths (i.e., for $30 \%$ of main-sequence dwarfs - see Lovis et al. 2009), because this kind of planets typically needs to have a bright $\mathrm{K}$ dwarf host to exceed the CoRoT detection threshold.

\section{Summary}

CoRoT has observed its first star field, IRa01, for 2 months since the beginning of 2008. It has obtained light curves of 3898 chromatic sources and 5974 monochromatic sources, which have been analysed by the detection teams. About one hundred sources have been classified as potential candidates and 50 of them have been kept as good candidates. The transit parameters of these candidates are listed in Table 3. About 40 of these should be followed-up with ground-based facilities. So far only two planets, CoRoT-1b and CoRoT-4b, have been confirmed, from IRa01, each published individually as the subject of a dedicated study. We provide in the Appendix a list of eclipsing binaries found in the field.

\section{References}

Aigrain, S., Collier Cameron, A., Ollivier, M., et al. 2008, A\&A, 488, L43 Aigrain, S., Pont, F., Fressin, F., et al. 2009, A\&A, 506, 425 
Auvergne, M., Bodin, P., Boisnard, L., et al. 2009, A\&A, 506, 411 Barge, P., Baglin, A., Auvergne, M., et al. 2008a, A\&A, 482, L17 Barge, P., Baglin, A., Auvergne, M., \& the CoRoT team. 2008b, in IAU Symp., 249,3

Carpano, S., \& Fridlund, M. 2008, A\&A, 485, 607

Deleuil, M., Meunier, J., Moutou, C., et al. 2009, AJ, 138, 649

Drummond, R., Lapeyrere, V., Auvergne, M., et al. 2008, A\&A, 487, 1209

Fressin, F., Guillot, T., Morello, V., \& Pont, F. 2007, A\&A, 475, 729

Gould, A., Dorsher, S., Gaudi, B. S., \& Udalski, A. 2006, Acta Astron., 56, 1

Kabath, P., Eigmüller, P., Erikson, A., et al. 2007, AJ, 134, 1560

Léger, A., Rouan, D., Schneider, J., et al. 2009, A\&A, 506, 287

Levenberg, K. 1944, The Quarterly of Applied Mathematics, 2, 164

Lovis, C., Mayor, M., Bouchy, F., et al. 2009, in IAU Symp., 253, 502

Marquardt, D. 1963, SIAM J. Appl. Math., 11, 431

Meunier, J.-C., Deleuil, M., Moutou, C., et al. 2007, in Astronomical Data Analysis Software and Systems XVI, ed. R. A. Shaw, F. Hill, \& D. J. Bell, ASP Conf. Ser., 376, 339
Moutou, C., Pont, F., Barge, P., et al. 2005, A\&A, 437, 355

Moutou, C., Aigrain, S., Almenara, J., et al. 2007, in Transiting Extrapolar Planets Workshop, ed. C. Afonso, D. Weldrake, \& T. Henning, ASP Conf. Ser., 366,127

Moutou, C., Pont, F., Bouchy, F., et al. 2009, A\&A, 506, 321

Pinheiro da Silva, L., Rolland, G., Lapeyrere, V., \& Auvergne, M. 2008, MNRAS, 384, 1337

Pont, F., Zucker, S., \& Queloz, D. 2006, MNRAS, 373, 231

Quentin, C. G., Barge, P., Cautain, R., et al. 2006, in ESA SP, ed. M. Fridlund, A. Baglin, J. Lochard, \& L. Conroy, ESA SP, 1306, 409

Régulo, C., Almenara, J. M., Alonso, R., Deeg, H., \& Roca Cortés, T. 2007, A\&A, 467, 1345

Renner, S., Rauer, H., Erikson, A., et al. 2008, A\&A, 492, 617

Seager, S., \& Mallén-Ornelas, G. 2003, ApJ, 585, 1038

Surace, C., Alonso, R., Barge, P., et al. 2008, SPIE Conf. Ser., 7019 


\section{Appendix A: Binary stars in CoRoT IRa01 field}

Table A.1 lists of all eclipsing binaries that have been identified in CoRoT-IRa01 field. Five of these sources (No. 4, 32, 34, 97, 123) were reported in Kabath et al. (2007) within their Berlin Exoplanet Search Telescope (BEST) survey of variable stars in the CoRoT fields. Sources 1 to 139 are ordinary eclipsing binaries (note that sources labeled 39 and 40 are two binaries in the same mask of CoRoT, so there is a single CoRoT identifier for both), whereas sources 140 to 145 are eclipsing binaries where only one eclipse has been found, so their period could not be determined (these are the so-called mono-transit events).

Table A.1. Eclipsing binary candidates found in IRa01.

\begin{tabular}{|c|c|c|c|c|c|c|c|c|c|}
\hline No. & CoRoT-ID & "Win-ID & Alpha $\left(^{\circ}\right)$ & $\overline{\operatorname{Delta}\left({ }^{\circ}\right)}$ & $\bar{V}$ Mag & $\overline{\text { Period (d) }}$ & $\begin{array}{l}\text { Epoch (d) } \\
+2454000\end{array}$ & $\overline{\overline{\text { Dur. (h) }}}$ & $\overline{\text { Depth (\%) }}$ \\
\hline 1 & 102759638 & E2 3724 & 101.248 & -2.61254 & 15.13 & $12.32900 \pm 1.00 \mathrm{E}-03$ & $142.39700 \pm 1.59 \mathrm{E}-01$ & 11.440 & $\overline{0.800}$ \\
\hline 2 & 102781577 & E2 3479 & 101.371 & -2.93473 & 15.33 & $5.00429 \pm 1.61 \mathrm{E}-02$ & $141.17440 \pm 1.17 \mathrm{E}-01$ & 7.200 & 0.300 \\
\hline 3 & 102819749 & E2 1568 & 101.639 & -2.59904 & 15.11 & $36.92300 \pm 1.00 \mathrm{E}-03$ & $162.36000 \pm 1.00 \mathrm{E}-02$ & 7.300 & 13.000 \\
\hline 4 & 102941623 & E2 2199 & 102.343 & -2.17165 & 15.31 & $1.65060 \pm 3.00 \mathrm{E}-04$ & $138.99400 \pm 3.00 \mathrm{E}-03$ & 5.900 & 0.270 \\
\hline 5 & 102707895 & E1 1873 & 100.951 & -0.96872 & 13.96 & $5.78390 \pm 5.00 \mathrm{E}-04$ & $136.85500 \pm 8.00 \mathrm{E}-03$ & 9.450 & 0.430 \\
\hline 6 & 102765395 & E2 4693 & 101.280 & -2.31180 & 15.88 & $1.57651 \pm 4.72 \mathrm{E}-04$ & $139.71463 \pm 7.22 \mathrm{E}-03$ & 1.730 & 0.630 \\
\hline 7 & 102855348 & E1 0081 & 10 & -1.20972 & 12.23 & $5.42500 \pm 2.50 \mathrm{E}-03$ & $136.77300 \pm 1.30 \mathrm{E}-02$ & 3.000 & 0.170 \\
\hline 8 & 102823343 & E1 4238 & 101.665 & -1.81256 & 15.57 & $7.44690 \pm 1.30 \mathrm{E}-03$ & $138.54600 \pm 2.00 \mathrm{E}-03$ & 3.620 & 6.800 \\
\hline 9 & 102771473 & E2 4752 & 101.315 & -2.24456 & 16.56 & $1.13230 \pm 2.46 \mathrm{E}-04$ & $139.37964 \pm 8.25 \mathrm{E}-03$ & 2.390 & 0.480 \\
\hline 10 & 102826074 & E1 4597 & 101.684 & -1.32652 & 16.25 & $48.57814 \pm 2.87 \mathrm{E}-03$ & $140.23557 \pm 1.87 \mathrm{E}-03$ & 10.310 & 15.620 \\
\hline 11 & 102725806 & E1 1875 & 101.057 & -1.58770 & 15.42 & $0.34180 \pm 1.00 \mathrm{E}-04$ & $136.02320 \pm 2.92 \mathrm{E}-03$ & 0.000 & 8.230 \\
\hline 12 & 102739450 & E1 2209 & 101.135 & -0.64769 & 15.62 & $2.07265 \pm 1.00 \mathrm{E}-05$ & $136.16830 \pm 6.83 \mathrm{E}-03$ & 0.560 & 2.820 \\
\hline 13 & 102760888 & E1 4417 & 101.255 & -1.70619 & 13.44 & $1.90450 \pm 1.90 \mathrm{E}-04$ & $136.09635 \pm 3.20 \mathrm{E}-03$ & 2.020 & 0.160 \\
\hline 14 & 102791304 & E2 4439 & 101.426 & -3.11075 & 16.30 & $13.57800 \pm 1.00 \mathrm{E}-03$ & $149.71745 \pm 7.30 \mathrm{E}-04$ & 25.200 & 49.000 \\
\hline 15 & 102794063 & E2 3276 & 101.442 & -2.80939 & 16.35 & $0.38194 \pm 5.00 \mathrm{E}-05$ & $138.34893 \pm 1.00 \mathrm{E}-04$ & 3.720 & 20.400 \\
\hline 16 & 102794135 & E1 0736 & 101.442 & -1.85562 & 14.63 & $0.26387 \pm 5.00 \mathrm{E}-05$ & $135.10625 \pm 1.00 \mathrm{E}-04$ & 1.710 & 5.760 \\
\hline 17 & 102798366 & E1 1488 & 101.476 & -1.25857 & 15.29 & $0.39568 \pm 5.00 \mathrm{E}-05$ & $135.47506 \pm 1.00 \mathrm{E}-04$ & 3.710 & 1.830 \\
\hline 18 & 102806220 & E2 3454 & 101.540 & -2.47491 & 15.92 & $0.34973 \pm 5.00 \mathrm{E}-04$ & $138.58722 \pm 1.00 \mathrm{E}-04$ & 3.700 & 35.000 \\
\hline 19 & 102806409 & E2 4376 & 101.542 & -3.14874 & 16.09 & $0.64735 \pm 5.00 \mathrm{E}-04$ & $138.54850 \pm 1.00 \mathrm{E}-04$ & 3.300 & 20.100 \\
\hline 20 & 102808511 & E2 0968 & 60 & -2.37561 & 13.37 & $0.23966 \pm 5.00 \mathrm{E}-04$ & $138.22310 \pm 1.0$ & 2.600 & 31.100 \\
\hline 21 & 102814334 & E1 1671 & & -1.5 & 14.12 & 0.657 & 760 & 3.300 & 0.190 \\
\hline 22 & 102819924 & E2 4711 & $101 .($ & -2.92284 & 16.17 & $16.70000 \pm 3.00 \mathrm{E}-03$ & $149.65103 \pm 2.6$ & 26.100 & 47.800 \\
\hline 23 & 102821683 & E2 2608 & 101 & -2.17193 & 14.75 & 1.8109 & 140.0311 & 18.700 & 2.110 \\
\hline 24 & 102822723 & E2 2726 & 101.661 & -2.26234 & 14.93 & $10.12150 \pm 3.55 \mathrm{E}-04$ & $144.23984 \pm 8.5$ & 11.200 & 30.400 \\
\hline 25 & 102826085 & E2 0778 & 101.684 & -2.24207 & 13.01 & $1.02583 \pm 7.00 \mathrm{E}-05$ & $138.88381 \pm 2.07 \mathrm{E}-03$ & 10.500 & 5.100 \\
\hline 26 & 102846142 & E1 1128 & 101.819 & -1.45863 & 15.04 & $0.41086 \pm 5.00 \mathrm{E}-04$ & $135.05939 \pm 1.00 \mathrm{E}-04$ & 4.600 & 14.500 \\
\hline 27 & 102870524 & E1 2045 & 101.963 & -1.48052 & 14.13 & $1.86800 \pm 1.00 \mathrm{E}-03$ & $136.47937 \pm 1.00 \mathrm{E}-03$ & 1.100 & 0.270 \\
\hline 28 & 102888076 & E2 3346 & 102.068 & -2.53040 & 15.37 & $24.19210 \pm 2.72 \mathrm{E}-03$ & $142.60754 \pm 3.00 \mathrm{E}-03$ & 5.900 & 34.100 \\
\hline 29 & 102897917 & E1 4302 & 102.120 & -0.72015 & 16.13 & $0.44560 \pm 5.00 \mathrm{E}-04$ & $135.27050 \pm 1.00 \mathrm{E}-04$ & 4.400 & 20.700 \\
\hline 30 & 102904593 & E1 1080 & 102.154 & -0.60466 & 15.13 & $16.89607 \pm 2.94 \mathrm{E}-04$ & $142.04979 \pm 3.40 \mathrm{E}-04$ & 5.500 & 17.900 \\
\hline 31 & 102910432 & E2 1277 & 102.185 & -2.62631 & 14.87 & $\pm 5.00 \mathrm{E}-04$ & 138.564 & 3.400 & 4.710 \\
\hline 32 & 102924081 & E2 0262 & 102.254 & -1.89538 & 12.27 & $0.37350 \pm 1.00 \mathrm{E}-04$ & $138.25600 \pm 1.00 \mathrm{E}-04$ & 4.000 & 24.100 \\
\hline 33 & 102939944 & E2 0738 & 34 & -2.1 & 14.36 & $\pm 5.00 \mathrm{E}-04$ & $\pm 5.00 \mathrm{E}-03$ & 2.200 & 0.240 \\
\hline 34 & 102940723 & E2 1704 & & -2.1 & 11.73 & $5.00 \mathrm{E}$ & 176 & 3.100 & 43.600 \\
\hline 35 & 1029 & E2 0915 & 10 & -2.3 & 13.21 & 20.1388 & 578 & 16.100 & 25.100 \\
\hline 36 & 1029 & E2 2 & & -2.0 & 15.81 & 04 & 04 & 4.300 & 30.500 \\
\hline 37 & 102844383 & E1 1495 & 10 & -1.3 & 15.10 & $1.5271 \varepsilon$ & 135.455 & 2.400 & 1.100 \\
\hline 38 & 102846496 & E2 2095 & 101.821 & -3.11782 & 14.77 & $5.00 \mathrm{E}-04$ & 138.4050 & 1.800 & 0.430 \\
\hline 39 & 102842572 & E2 2746 & 101.795 & -2.05667 & 15.91 & $4.00256 \pm 5.00 \mathrm{E}-04$ & $138.17830 \pm 5.00 \mathrm{E}-04$ & 3.300 & 9.900 \\
\hline 40 & - & - & - & - & - & $2.46350 \pm 5.00 \mathrm{E}-04$ & $139.19670 \pm 5.00 \mathrm{E}-04$ & 2.500 & 2.700 \\
\hline 41 & 102745492 & E1 4732 & 168 & -1.12 & 16.27 & $3.60467 \pm 7.64 \mathrm{E}-04$ & $138.95489 \pm 7.50 \mathrm{E}-03$ & 2.472 & 5.285 \\
\hline 42 & 102901962 & E1 3608 & 102.141 & -1.68750 & 16.41 & $2.44483 \pm 5.17 \mathrm{E}-03$ & $137.09217 \pm 4.55 \mathrm{E}-02$ & 4.174 & 8.865 \\
\hline 43 & 102912741 & E2 0254 & 102.196 & -2.89809 & 13.35 & $1.24542 \pm 8.90 \mathrm{E}-05$ & $138.51084 \pm 2.27 \mathrm{E}-03$ & 2.117 & 9.565 \\
\hline 44 & 102817472 & E2 1390 & 101.623 & -2.75731 & 15.13 & $1.47876 \pm 3.48 \mathrm{E}-04$ & $138.57094 \pm 7.04 \mathrm{E}-03$ & 4.702 & 0.575 \\
\hline 45 & 102846132 & E2 3660 & 101.819 & -2.49772 & 16.35 & $1.38144 \pm 5.24 \mathrm{E}-04$ & $139.38388 \pm 6.70 \mathrm{E}-03$ & 4.487 & 8.665 \\
\hline 46 & 102853429 & E2 1218 & 101.865 & -2.79528 & 13.53 & $1.63806 \pm 1.16 \mathrm{E}-04$ & $138.50031 \pm 2.21 \mathrm{E}-03$ & 3.141 & 1.550 \\
\hline 47 & 102879429 & E1 1827 & 102.018 & -1.57061 & 15.48 & $4.03054 \pm 6.90 \mathrm{E}-05$ & $137.84405 \pm 1.12 \mathrm{E}-03$ & 2.872 & 6.560 \\
\hline 48 & 102982347 & E2 1745 & 102.563 & -2.97465 & 13.94 & $2.97762 \pm 4.30 \mathrm{E}-05$ & $139.86651 \pm 4.16 \mathrm{E}-04$ & 4.113 & 10.770 \\
\hline 49 & 102756466 & E1 3987 & 101.230 & -0.86304 & 15.48 & $16.76987 \pm 2.93 \mathrm{E}-01$ & $151.94809 \pm 1.26 \mathrm{E}-01$ & 6.752 & 6.785 \\
\hline 50 & 102779171 & E1 1499 & 101.357 & -1.06390 & 13.44 & $11.33939 \pm 3.72 \mathrm{E}-02$ & $143.75596 \pm 1.34 \mathrm{E}-01$ & 7.545 & 2.000 \\
\hline
\end{tabular}


Table A.1. continued.

\begin{tabular}{|c|c|c|c|c|c|c|c|c|c|}
\hline No. & CoRoT-ID & "Win-ID & Alpha $\left(^{\circ}\right)$ & $\overline{\text { Delta }\left({ }^{\circ}\right)}$ & $\overline{\overline{V \text { Mag }}}$ & $\overline{\text { Period (d) }}$ & $\begin{array}{l}\text { Epoch (d) } \\
+2454000\end{array}$ & $\overline{\text { Dur. (h) }}$ & $\overline{\text { Depth (\%) }}$ \\
\hline 51 & 102738614 & E1 0827 & 101.130 & -1.17246 & 14.45 & $7.76844 \pm 5.16 \mathrm{E}-02$ & $135.81384 \pm 1.46 \mathrm{E}-01$ & 3.511 & 17.555 \\
\hline 52 & 102818537 & E2 2620 & 101.631 & -2.85605 & 14.45 & $2.27296 \pm 4.80 \mathrm{E}-05$ & $139.72413 \pm 6.41 \mathrm{E}-04$ & 5.438 & 9.254 \\
\hline 53 & 102820928 & E2 4500 & 101.648 & -2.61561 & 16.16 & $1.82502 \pm 1.77 \mathrm{E}-04$ & $138.45024 \pm 3.15 \mathrm{E}-03$ & 3.848 & 4.915 \\
\hline 54 & 102867757 & E2 4431 & 101.947 & -2.68001 & 16.11 & $2.68580 \pm 2.89 \mathrm{E}-04$ & $140.21954 \pm 3.46 \mathrm{E}-03$ & 2.552 & 8.510 \\
\hline 55 & 102708916 & E1 0484 & 100.957 & -0.79757 & 13.97 & $6.18906 \pm 6.35 \mathrm{E}-02$ & $141.10739 \pm 3.44 \mathrm{E}-01$ & 5.335 & 21.340 \\
\hline 56 & 102726405 & E1 0801 & 101.061 & -1.37603 & 12.76 & $2.54204 \pm 2.90 \mathrm{E}-05$ & $138.05727 \pm 3.47 \mathrm{E}-04$ & 4.280 & 18.720 \\
\hline 57 & 102732394 & E1 1251 & 101.095 & -1.42804 & 14.96 & $1.57360 \pm 4.30 \mathrm{E}-05$ & $135.74521 \pm 9.19 \mathrm{E}-04$ & 3.056 & 6.810 \\
\hline 58 & 102733170 & E1 1543 & 101.100 & -1.61003 & 13.68 & $1.97940 \pm 4.50 \mathrm{E}-05$ & $135.86023 \pm 7.68 \mathrm{E}-04$ & 3.949 & 3.770 \\
\hline 59 & 102734453 & E1 2507 & 101.107 & -0.67484 & 14.47 & $8.37056 \pm 6.80 \mathrm{E}-05$ & $144.18882 \pm 3.35 \mathrm{E}-03$ & 21.700 & .371 \\
\hline 60 & 102735868 & E1 3810 & 101.115 & -1.33074 & 15.25 & $1.64709 \pm 1.80 \mathrm{E}-05$ & $135.56796 \pm 2.15 \mathrm{E}-03$ & 3.775 & 9.000 \\
\hline 61 & 102741994 & E1 2336 & 101.149 & -1.64399 & 14.47 & $4.62211 \pm 1.07 \mathrm{E}-04$ & $135.78605 \pm 7.22 \mathrm{E}-04$ & 4.124 & 9.500 \\
\hline 62 & 102752408 & E1 3080 & 101.207 & -1.12243 & 16.13 & $21.26057 \pm 5.41 \mathrm{E}-03$ & $146.95066 \pm 6.96 \mathrm{E}-03$ & 4.600 & 1.525 \\
\hline 63 & 102754263 & E1 3846 & 101.217 & -1.05010 & 15.31 & $2.45716 \pm 1.50 \mathrm{E}-04$ & $136.94466 \pm 1.78 \mathrm{E}-03$ & 2.994 & 7.260 \\
\hline 64 & 102756903 & E1 4392 & 101.232 & -1.44495 & 15.90 & $0.97909 \pm 1.50 \mathrm{E}-05$ & $136.17055 \pm 4.74 \mathrm{E}-04$ & 2.418 & 3.070 \\
\hline 65 & 102757626 & E1 0791 & 101.236 & -1.23750 & 14.70 & $1.20543 \pm 6.10 \mathrm{E}-05$ & $135.91488 \pm 1.60 \mathrm{E}-03$ & 3.441 & 9.600 \\
\hline 66 & 102764398 & E2 3602 & 101.275 & -2.81121 & 16.23 & $0.92744 \pm 5.00 \mathrm{E}-04$ & $138.08047 \pm 1.00 \mathrm{E}-04$ & 2.702 & 4.689 \\
\hline 67 & 102768859 & E2 4148 & 101.300 & -2.81762 & 16.10 & $8.06342 \pm 5.26 \mathrm{E}-04$ & $141.27802 \pm 5.80 \mathrm{E}-03$ & 4.913 & 1.444 \\
\hline 68 & 102773399 & E1 2875 & 101.326 & -0.95011 & 14.81 & $0.60560 \pm 1.00 \mathrm{E}-04$ & $135.51522 \pm 7.15 \mathrm{E}-03$ & 2.702 & 3.850 \\
\hline 69 & 102774523 & E1 1052 & 101.332 & -1.85324 & 14.89 & $5.91776 \pm 1.09 \mathrm{E}-04$ & $135.67799 \pm 5.51 \mathrm{E}-04$ & 3.901 & 27.240 \\
\hline 70 & 102776173 & E2 1176 & 101.341 & -3.21882 & 14.90 & $0.72508 \pm 5.00 \mathrm{E}-05$ & $138.59908 \pm 5.00 \mathrm{E}-04$ & 2.702 & 3.240 \\
\hline 71 & 102776386 & E2 1137 & 101.342 & -2.86191 & 13.50 & $2.20677 \pm 2.70 \mathrm{E}-04$ & $139.42573 \pm 3.74 \mathrm{E}-03$ & 3.684 & 3.570 \\
\hline 72 & 102776565 & E2 2143 & 101.343 & -3.15951 & 14.63 & $2.20584 \pm 6.50 \mathrm{E}-05$ & $140.76363 \pm 8.51 \mathrm{E}-04$ & 4.111 & 56.400 \\
\hline 73 & 1027 & E1 3 & 14 & -0.6 & 16.23 & $2.18327 \pm$ & $38286 \pm$ & 4.109 & 59.790 \\
\hline 74 & 1027 & E1 1002 & 10 & -0.66941 & 14.88 & $78215 \pm 1$ & $29534 \pm$ & 2.702 & 0.430 \\
\hline 75 & 1027 & E1 2613 & 101.394 & -1.57614 & 15.88 & $4.71634 \pm$ & $139.61223 \pm$ & 5.267 & 23.860 \\
\hline 76 & $1027 \mathrm{c}$ & E2 1005 & 101.421 & -2.46922 & 14.72 & $4.91014=$ & $139.89439 \pm$ & .419 & 15.410 \\
\hline 77 & 102793963 & E1 3124 & 101.441 & -1.62531 & 16.19 & $1.24225 \pm 1.00 \mathrm{E}-04$ & $135.40128 \pm 5.95 \mathrm{E}-04$ & 1.596 & 2.070 \\
\hline 78 & 102802054 & E2 4445 & 101.506 & -2.33150 & 15.96 & $2.12884 \pm 1.69 \mathrm{E}-04$ & $140.44080 \pm 2.20 \mathrm{E}-03$ & .540 & 2.440 \\
\hline 79 & 102803023 & E1 4206 & 101.514 & -0.64875 & 15.69 & $2.32061 \pm 6.00 \mathrm{E}-05$ & $137.82606 \pm 8.25 \mathrm{E}-04$ & .263 & 15.620 \\
\hline 80 & 102806377 & E2 0836 & 101.541 & -2.03210 & 13.13 & $3.81666 \pm 3.70 \mathrm{E}-03$ & $142.04834 \pm 2.16 \mathrm{E}-02$ & 4.222 & 13.380 \\
\hline 81 & 10280 & E2 1918 & .543 & -3.23352 & 14.24 & $3.66704 \pm 3$ & $140.43028 \pm 2.57 \mathrm{E}-03$ & 925 & 16.500 \\
\hline 82 & 102809393 & E2 0486 & 101.566 & -2.83432 & 14.08 & $7.71063 \pm 4.54 \mathrm{E}-02$ & $139.08695 \pm 5.58 \mathrm{E}-02$ & 6.247 & 7.470 \\
\hline 83 & 10281 & E2 0416 & 101.582 & -1.98315 & 12.47 & $1.66868 \pm 1.25 \mathrm{E}-04$ & $139.03337 \pm 2.25 \mathrm{E}-03$ & 2.782 & 2.800 \\
\hline 84 & 1028 & E1 4561 & 101.592 & -0.99226 & 16.24 & $1.30626 \pm 3.90 \mathrm{E}-05$ & $136.71588 \pm 9.15 \mathrm{E}-04$ & 3.026 & 23.100 \\
\hline 85 & 1028 & E2 2 & 613 & -2.31198 & 15.63 & $7.44703 \pm 2.65 \mathrm{E}-04$ & $140.40955 \pm 8.9$ & 6.238 & 25.550 \\
\hline 86 & 102818428 & E2 1307 & & -2.2 & 13.79 & $7.45491 \pm 4$ & $142.37746 \pm 1.54 \mathrm{E}-03$ & 6.238 & 5.520 \\
\hline 87 & 10281 & E2 3 & & -2.8 & 15.15 & $596 \pm$ & -03 & 12 & 3.730 \\
\hline 88 & 102 & $\mathrm{E}$ & & -1.5 & 15.86 & 05 & 04 & 3.462 & 30.170 \\
\hline 89 & 1028 & E1 1 & 10 & -0.7 & 14.28 & 8.09754 & $9042 \pm$ & 6.403 & 22.900 \\
\hline 90 & 10282 & E1 3 & 10 & -0.68487 & 15.10 & $1.47677 \pm$ & $13 r$ & 3.046 & 26.680 \\
\hline 91 & 102828417 & E2 1036 & 101.701 & -2.04626 & 14.80 & $9.59460=$ & $147.32365 \pm 7.2$ & 6.453 & 14.340 \\
\hline 92 & 102835452 & E2 4071 & 101.748 & -2.22388 & 15.71 & $6.93290 \pm 7.68 \mathrm{E}-03$ & $139.00417 \pm 1.69 \mathrm{E}-02$ & 5.080 & 50.780 \\
\hline 93 & 102836138 & E1 0844 & 101.753 & -1.34797 & 14.72 & $3.55818 \pm 4.90 \mathrm{E}-05$ & $137.20937 \pm 3.67 \mathrm{E}-04$ & 3.922 & 16.770 \\
\hline 94 & 102836169 & E2 4009 & .753 & -2.56595 & 16.16 & $1.18554 \pm 5.00 \mathrm{E}-05$ & $139.23407 \pm 1.40 \mathrm{E}-03$ & 1.874 & 24.690 \\
\hline 95 & 102840080 & E2 3619 & 779 & -2.93765 & 15.38 & $2.33737 \pm 1.93 \mathrm{E}-04$ & $139.61448 \pm 2.40 \mathrm{E}-03$ & 2.557 & 2.180 \\
\hline 96 & 102841939 & E1 5038 & 101.791 & -0.55531 & 16.02 & $2.37762 \pm 3.60 \mathrm{E}-05$ & $135.82534 \pm 4.82 \mathrm{E}-04$ & 3.983 & 29.670 \\
\hline 97 & 102842120 & E2 3952 & 101.792 & -2.95054 & 14.17 & $1.10449 \pm 3.90 \mathrm{E}-05$ & $138.62653 \pm 1.13 \mathrm{E}-03$ & 3.285 & 38.860 \\
\hline 98 & 102842466 & E1 3571 & 101.795 & -1.03743 & 14.42 & $4.91740 \pm 5.04 \mathrm{E}-04$ & $138.74857 \pm 2.86 \mathrm{E}-03$ & 5.419 & 67.290 \\
\hline 99 & 102844991 & E1 3252 & 101.812 & -0.91820 & 15.99 & $1.07425 \pm 1.80 \mathrm{E}-05$ & $135.53889 \pm 5.45 \mathrm{E}-04$ & 2.428 & 15.620 \\
\hline 100 & 102849348 & E2 2452 & 101.840 & -2.82048 & 14.79 & $1.81837 \pm 5.60 \mathrm{E}-05$ & $138.60443 \pm 9.44 \mathrm{E}-04$ & 3.223 & 10.440 \\
\hline 101 & 1028 & E2 3081 & & -2.25781 & 15.67 & $1.01078 \pm 2.10 \mathrm{E}-05$ & $139.00262 \pm 6$ & .419 & 22.270 \\
\hline 102 & 102852229 & E2 0872 & 101.858 & -2.77432 & 13.31 & $6.06061 \pm 3.82 \mathrm{E}-02$ & $140.73996 \pm 1.83 \mathrm{E}-01$ & 5.756 & 9.680 \\
\hline 103 & 102858100 & E2 2099 & 101.892 & -3.07796 & 14.46 & $1.31134 \pm 9.00 \mathrm{E}-05$ & $138.64401 \pm 2.12 \mathrm{E}-03$ & 3.311 & 4.330 \\
\hline 104 & 102870155 & E2 4907 & 101.961 & -2.81992 & 16.20 & $2.78294 \pm 8.40 \mathrm{E}-05$ & $139.98194 \pm 8.08 \mathrm{E}-04$ & 4.440 & 15.050 \\
\hline 105 & 102870613 & E2 0117 & 101.964 & -2.68863 & 12.54 & $7.13947 \pm 4.85 \mathrm{E}-04$ & $145.98116 \pm 1.75 \mathrm{E}-03$ & 6.084 & 41.650 \\
\hline 106 & 102870852 & E2 0609 & 101.965 & -2.90340 & 14.26 & $0.76471 \pm 5.20 \mathrm{E}-04$ & $138.56904 \pm 1.89 \mathrm{E}-03$ & 2.844 & 2.500 \\
\hline 107 & 102872646 & E2 0818 & 101.976 & -2.72664 & 14.40 & $1.88286 \pm 4.17 \mathrm{E}-04$ & $138.92797 \pm 6.80 \mathrm{E}-05$ & 3.940 & 4.350 \\
\hline 108 & & E2 0365 & 2.017 & -2.09075 & 13.67 & $0.97728 \pm 5.00 \mathrm{E}-04$ & $138.77715 \pm 3.43 \mathrm{E}-03$ & 3.129 & 6.730 \\
\hline 109 & 102882044 & E1 3079 & 102.033 & -0.48467 & 16.08 & $9.07345 \pm 5.60 \mathrm{E}-05$ & $136.91781 \pm 1.80 \mathrm{E}-05$ & 4.127 & 28.910 \\
\hline
\end{tabular}


Table A.1. continued.

\begin{tabular}{|c|c|c|c|c|c|c|c|c|c|}
\hline No. & "CoRoT-ID & $\overline{\overline{\text { Win-ID }}}$ & $\overline{\text { Alpha }\left({ }^{\circ}\right)}$ & $\overline{\overline{\text { Delta }\left(^{\circ}\right)}}$ & $\overline{\overline{V \text { Mag }}}$ & $\overline{\overline{\text { Period }(\mathrm{d})}}$ & $\begin{array}{l}\text { Epoch (d) } \\
+2454000\end{array}$ & $\overline{\overline{\text { Dur. (h) }}}$ & $\overline{\overline{\text { Depth }(\%)}}$ \\
\hline 110 & 102884662 & E1 1938 & 102.048 & -1.00089 & 15.93 & $3.84822 \pm 2.16 \mathrm{E}-04$ & $138.97463 \pm 1.73 \mathrm{E}-03$ & 4.935 & 35.770 \\
\hline 111 & 102886012 & E1 4690 & 102.056 & -1.61420 & 16.34 & $1.58466 \pm 2.52 \mathrm{E}-04$ & $136.15763 \pm 4.04 \mathrm{E}-03$ & 2.631 & 0.810 \\
\hline 112 & 102889458 & E1 4646 & 102.075 & -1.00521 & 16.30 & $2.01989 \pm 3.30 \mathrm{E}-05$ & $136.31380 \pm 5.05 \mathrm{E}-04$ & 3.953 & 22.100 \\
\hline 113 & 102892869 & E1 3024 & 102.093 & -0.56110 & 15.08 & $4.07590 \pm 6.59 \mathrm{E}-04$ & $139.53177 \pm 5.41 \mathrm{E}-03$ & 3.810 & 1.589 \\
\hline 114 & 102896719 & E1 3444 & 102.114 & -0.47842 & 14.90 & $1.23114 \pm 1.10 \mathrm{E}-04$ & $135.97699 \pm 2.98 \mathrm{E}-03$ & 3.444 & 2.090 \\
\hline 115 & 102900859 & E1 1220 & 102.135 & -0.53002 & 13.42 & $4.85346 \pm 1.48 \mathrm{E}-04$ & $136.52903 \pm 8.29 \mathrm{E}-04$ & 5.416 & 11.250 \\
\hline 116 & 102902696 & E1 1276 & 102.145 & -0.75803 & 13.58 & $1.98087 \pm 1.40 \mathrm{E}-05$ & $136.66216 \pm 2.41 \mathrm{E}-04$ & 3.665 & 26.510 \\
\hline 117 & 102914654 & E2 4083 & 102.206 & -2.15859 & 15.95 & $1.20531 \pm 1.80 \mathrm{E}-05$ & $139.12553 \pm 4.68 \mathrm{E}-04$ & 3.441 & 20.550 \\
\hline 118 & 102929837 & E2 1064 & 102.283 & -2.89422 & 14.82 & $3.81996 \pm 4.10 \mathrm{E}-05$ & $139.41564 \pm 3.47 \mathrm{E}-04$ & 4.934 & 37.900 \\
\hline 119 & 102930316 & E2 2382 & 102.286 & -2.95754 & 15.74 & $1.49577 \pm 1.26 \mathrm{E}-04$ & $139.76033 \pm 2.53 \mathrm{E}-03$ & 3.617 & 5.110 \\
\hline 120 & 102931335 & E1 3946 & 102.291 & -1.44787 & 15.47 & $3.97923 \pm 2.90 \mathrm{E}-05$ & $138.84505 \pm 2.29 \mathrm{E}-04$ & 4.516 & 25.390 \\
\hline 121 & 102932176 & E2 1219 & 102.295 & -1.99175 & 13.71 & $0.87225 \pm 1.30 \mathrm{E}-05$ & $139.05420 \pm 2.29 \mathrm{E}-04$ & 2.987 & 21.190 \\
\hline 122 & 102943073 & E2 0151 & 102.349 & -2.48166 & 12.88 & $1.64410 \pm 7.50 \mathrm{E}-05$ & $139.62393 \pm 1.34 \mathrm{E}-03$ & 2.921 & 4.320 \\
\hline 123 & 102955089 & E2 1261 & 102.408 & -1.83831 & 14.95 & $0.57161 \pm 1.70 \mathrm{E}-05$ & $138.98060 \pm 3.17 \mathrm{E}-04$ & 2.702 & 30.420 \\
\hline 124 & 102961237 & E2 3896 & 102.439 & -2.56702 & 15.58 & $1.02102 \pm 6.40 \mathrm{E}-05$ & $139.41378 \pm 1.77 \mathrm{E}-03$ & 3.132 & 4.220 \\
\hline 125 & 102965963 & E2 4756 & 102.467 & -2.16127 & 15.71 & $1.90365 \pm 3.30 \mathrm{E}-05$ & $140.09132 \pm 4.99 \mathrm{E}-04$ & 3.942 & 16.660 \\
\hline 126 & 102980178 & E2 4236 & 102.550 & -2.68934 & 15.73 & $5.05476 \pm 1.04 \mathrm{E}-03$ & $139.07148 \pm 4.98 \mathrm{E}-03$ & 5.426 & 46.710 \\
\hline 127 & 102983538 & E2 2825 & 102.570 & -2.93102 & 15.01 & $1.45435 \pm 1.20 \mathrm{E}-04$ & $139.03886 \pm 2.39 \mathrm{E}-03$ & 3.612 & 6.330 \\
\hline 128 & 102801922 & E1 0617 & 101.505 & -1.50686 & 14.43 & $5.45907 \pm 4.20 \mathrm{E}-05$ & $138.93015 \pm 2.32 \mathrm{E}-03$ & 3.503 & 0.895 \\
\hline 129 & 102927840 & E2 4136 & 102.273 & -2.66893 & 14.56 & $10.29535 \pm 8.58 \mathrm{E}-03$ & $140.69140 \pm 1.81 \mathrm{E}-02$ & 7.055 & 0.600 \\
\hline 130 & 102726103 & E1 0830 & 101.060 & -1.22901 & 14.54 & $3.81466 \pm 2.20 \mathrm{E}-05$ & $137.99633 \pm 1.82 \mathrm{E}-03$ & 1.894 & 0.565 \\
\hline 131 & 102786821 & E1 2938 & 101.400 & -1.32339 & 14.71 & $1.91876 \pm 1.88 \mathrm{E}-04$ & $135.51978 \pm 3.41 \mathrm{E}-03$ & 2.969 & 0.730 \\
\hline 132 & 102802298 & E2 4626 & 101.508 & -2.45497 & 15.51 & $3.71918 \pm 5.20 \mathrm{E}-05$ & $139.05857 \pm 4.08 \mathrm{E}-04$ & 3.578 & 10.590 \\
\hline 133 & 102932955 & E2 2046 & 102.299 & -2.76750 & 15.49 & $24.25906 \pm 4.75 \mathrm{E}-04$ & $140.53257 \pm 5.33 \mathrm{E}-04$ & 3.960 & 6.960 \\
\hline 134 & 102806484 & E2 4826 & 101.542 & -2.71639 & 16.18 & $9.56081 \pm 4.90 \mathrm{E}-05$ & $140.53819 \pm 1.38 \mathrm{E}-03$ & 2.729 & 1.585 \\
\hline 135 & 102735257 & E1 3236 & 101.111 & -1.635940 & 16.12 & $23.6935 \pm 5.00 \mathrm{e}-04$ & $156.952 \pm 5.00 \mathrm{e}-04$ & 4.265 & 11.6 \\
\hline 136 & 102937382 & E2 4533 & 102.322 & -1.909260 & 15.02 & $7.69724 \pm 3.89 \mathrm{E}-03$ & $139.15290 \pm 1.38 \mathrm{E}-03$ & 6.247 & 0.170 \\
\hline 137 & 102734591 & E1 0663 & 101.107 & -1.291500 & 14.39 & $8.16270 \pm 6.13 \mathrm{E}-03$ & $139.34211 \pm 2.35 \mathrm{E}-02$ & 6.400 & 0.100 \\
\hline 138 & 102751150 & E2 0193 & 101.200 & -2.087960 & 13.07 & $6.34015 \pm 1.50 \mathrm{E}-03$ & $144.05301 \pm 6.23 \mathrm{E}-03$ & 5.910 & 0.160 \\
\hline 139 & 102805003 & E2 2539 & 101.530 & -1.548410 & 14.54 & $5.57236 \pm 6.45 \mathrm{E}-03$ & $136.21190 \pm 2.65 \mathrm{E}-03$ & 13.800 & 0.110 \\
\hline 140 & 102765275 & E1 2060 & 101.280 & -0.671730 & 15.55 & - & $177.43000 \pm 1.00 \mathrm{E}-02$ & 7.200 & 6.200 \\
\hline 141 & 102829388 & E2 3914 & 101.708 & -3.030360 & 15.50 & - & $163.86000 \pm 1.00 \mathrm{E}-02$ & 18.400 & 8.500 \\
\hline 142 & 102855409 & E2 1633 & 101.877 & -2.299030 & 13.18 & - & $155.43000 \pm 1.00 \mathrm{E}-02$ & 20.260 & 17.200 \\
\hline 143 & 102868004 & E2 2416 & 101.949 & -2.206550 & 15.72 & - & $172.54000 \pm 1.00 \mathrm{E}-02$ & 5.750 & 5.700 \\
\hline 144 & 102919036 & E1 4818 & 102.229 & -1.072490 & 15.69 & - & $174.45000 \pm 1.00 \mathrm{E}-02$ & 17.800 & 1.800 \\
\hline 145 & 102801672 & E2 4912 & 101.503 & -2.599640 & 16.34 & - & $138.18000 \pm 1.00 \mathrm{E}-02$ & 9.070 & 8.200 \\
\hline
\end{tabular}

